\title{
Two-Dimensional Orthogonal Filter Banks with Directional Vanishing Moments
}

\author{
Jianping Zhou and Minh N. Do \\ Department of Electrical and Computer Engineering \\ University of Illinois at Urbana-Champaign, Urbana, IL 61801, USA
}

\begin{abstract}
We present two-dimensional filter banks with directional vanishing moments. The directional-vanishing-moment condition is crucial for the regularity of directional filter banks. However, it is a challenging task to design orthogonal filter banks with directional vanishing moments. Due to the lack of multidimensional factorization theorems, traditional one-dimensional methods cannot be extended to higher dimensional cases. Kovačević and Vetterli investigated the design of two-dimensional orthogonal filter banks and proposed a set of closed-form solutions called the lattice structure, where the polyphase matrix of the filter bank is characterized with a set of rotation parameters. Orthogonal filter banks with lattice structures have simple implementation. We propose a method of designing orthogonal filter banks with directional vanishing moments based on this lattice structure. The constraint of directional vanishing moments is imposed on the rotation parameters. We find the solutions of rotation parameters have special structure. Based on this structure, we find the closed-form solution for orthogonal filter banks with directional vanishing moments.
\end{abstract}

Keywords: d irectional vanishing moments, filter banks, geometric and directional analysis, lattice structure, multidimensional, orthogonal, nonseparable filter design, polyphase.

\section{INTRODUCTION}

Wavelets and filter banks have achieved great success in signal processing and communications applications. Wavelet transforms provide a multiresolution representation that captures the discontinuity locally in the signal. Moreover, wavelets provide better nonlinear approximation, which implies better performance in data compression and processing $[1,2]$. The decay rate of wavelet coefficients is $O\left(N^{-1}\right)$ for piecewise smooth signals, while that of Fourier coefficients is $O\left(N^{-1 / 2}\right)$. Vanishing moments play a key role in the design of wavelets and filter banks $[2,3]$.

In recent years, geometric image representation has received more and more interest [4-9]. Olshausen and Field showed that directionality plays a key role in human visual system [10]. Bamberger proposed the critically sampled directional filter banks which decompose images into different directional subbands [4]. Based on directional filter banks, Do and Vetterli proposed an efficient directional multiresolution image representation called the contourlet transform [9]. For efficient representation, both directional filter banks and the contourlet transform require directional vanishing moments (DVM), which are defined as vanishing moments along a specific direction. Cunha and Do characterized and designed biorthogonal filter banks with DVM [11]. However, there is no existing literature addressing the design of orthogonal filter banks with DVM.

Orthogonal filter banks have been widely used in multirate applications due to their useful properties such as energy preservation. The design problem leads to spectral factorization. Since there is no multidimensional factorization theorem, designing multidimensional orthogonal filter banks is challenging. Kovačević and Vetterli designed a class of orthogonal filter banks based on the lattice structure, which is characterized by a set of rotation parameters [12]. This class of orthogonal filter banks is easy to implement and is a general subset of orthogonal filter banks. Stanhill and Zeevi investigated the design of two-dimensional lattice orthogonal filter

Further author information: (Send correspondence to Jianping Zhou)

Jianping Zhou: E-mail: jzhou2@uiuc.edu

Minh Do: E-mail: minhdo@uiuc.edu 
banks with vanishing moments in [13], but they do not consider directional vanishing moments, which are more difficult.

Imposing directional vanishing moments on a lattice orthogonal filter bank leads to a set of complex nonlinear equations with respect to these parameters, which is difficult to solve. In this work, we propose a complete characterization of lattice orthogonal filter banks with directional vanishing moments. The DVM constraints are imposed on the rotation parameters. We find the solutions of rotation parameters have special structure. Based on this structure, we find a closed-form solution for the orthogonal filter banks with DVM. The rest of this paper is organized as follows. We introduce orthogonal filter banks and lattice structures in Section 2 . In Section 3 , we characterize orthogonal filter banks with DVM and propose explicit formulae for orthogonal filter banks with first-order DVM. Then we extend this result to orthogonal filter banks with higher-order DVM in Section 4 . We draw conclusions in Section 5. Due to the space limitation, we only present the results in this paper. Please refer to $[14]$ for proofs.

\section{ORTHOGONAL FILTER BANKS AND LATTICE STRUCTURES}

We start with notation. We let $\boldsymbol{z}$ stand for a two-dimensional (2-D) variable $\boldsymbol{z}=\left[z_{1}, z_{2}\right]^{T}$ and $\boldsymbol{z}^{-1}$ stand for $\left[z_{1}^{-1}, z_{2}^{-1}\right]^{T}$. Raising $\boldsymbol{z}$ to a 2 -D integer vector power $\boldsymbol{k}=\left[k_{1}, k_{2}\right]^{T}$ yields $\boldsymbol{z}^{\boldsymbol{k}}=z_{1}^{k_{1}} z_{2}^{k_{1}}$.

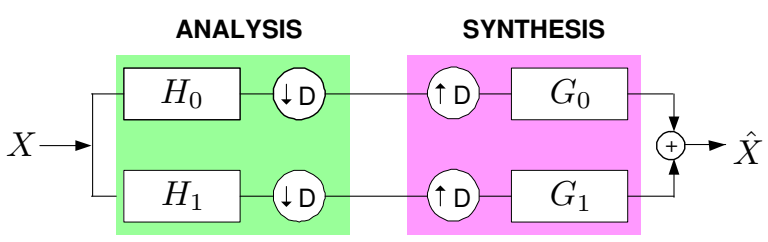

(a)

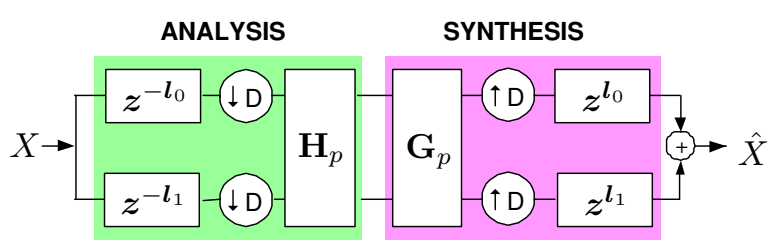

(b)

Figure 1. Two-dimensional filter banks and polyphase representation. (a) A two-dimensional two-channel filter bank: $H_{i}$ and $G_{i}$ are analysis and synthesis filters, respectively; $\mathbf{D}$ is a $2 \times 2$ sampling matrix. (b) Polyphase representation: $\mathbf{H}_{p}$ and $\mathbf{G}_{p}$ are $2 \times 2$ analysis and synthesis polyphase matrices, respectively; $\boldsymbol{l}_{0}$ and $\boldsymbol{l}_{1}$ are integer vectors of the form $\mathbf{D} \boldsymbol{t}$, such that $\boldsymbol{t} \in[0,1)^{2}$.

Consider a 2-D two-channel filter bank as shown in Fig. 1(a). For implementation purposes, we only consider filter banks with rational filters. We are interested in the critically sampled filter bank in which the sampling rate is equal to the number of channels, that is, $|\operatorname{det} \mathbf{D}|=2$. In the polyphase domain, the analysis and synthesis parts can be represented by $2 \times 2$ polyphase matrices $\mathbf{H}_{p}(\boldsymbol{z})$ and $\mathbf{G}_{p}(\boldsymbol{z})$, respectively, as shown in Fig. 1(b). The analysis and synthesis filters are related to the corresponding polyphase matrices as

$$
\begin{aligned}
& \left(\begin{array}{l}
H_{0}(\boldsymbol{z}) \\
H_{1}(\boldsymbol{z})
\end{array}\right)=\mathbf{H}_{p}\left(\boldsymbol{z}^{\boldsymbol{d}_{1}}, \boldsymbol{z}^{\boldsymbol{d}_{2}}\right)\left(\begin{array}{l}
\boldsymbol{z}^{-\boldsymbol{l}_{0}} \\
\boldsymbol{z}^{-\boldsymbol{l}_{1}}
\end{array}\right), \\
& \left(\begin{array}{ll}
\left.G_{0}(\boldsymbol{z}) \quad G_{1}(\boldsymbol{z})\right)=\left(\begin{array}{ll}
\boldsymbol{z}^{\boldsymbol{l}_{0}} & \boldsymbol{z}^{\boldsymbol{l}_{1}}
\end{array}\right) \mathbf{G}_{p}\left(\boldsymbol{z}^{\boldsymbol{d}_{1}}, \boldsymbol{z}^{\boldsymbol{d}_{2}}\right.
\end{array}\right),
\end{aligned}
$$

where $\boldsymbol{d}_{1}$ and $\boldsymbol{d}_{2}$ are columns of $\mathbf{D}$, and $\boldsymbol{l}_{0}$ and $\boldsymbol{l}_{1}$ are integer vectors of the form $\mathbf{D} \boldsymbol{t}$, such that $\boldsymbol{t} \in[0,1)^{2}[15](\mathrm{pp}$. $561-566)$.

In the polyphase domain, the perfect reconstruction condition $\hat{X}(\boldsymbol{z})=X(\boldsymbol{z})$ is equivalent to $\mathbf{H}_{p}(\boldsymbol{z}) \mathbf{G}_{p}(\boldsymbol{z})=\mathbf{I}$, where $\mathbf{I}$ is the $2 \times 2$ identity matrix. Orthogonal filter banks additionally require $\mathbf{H}_{p}(\boldsymbol{z})=\mathbf{G}_{p}^{T}\left(\boldsymbol{z}^{-1}\right)$. Therefore, the synthesis polyphase matrix $\mathbf{G}_{p}(\boldsymbol{z})$ satisfies $\mathbf{G}_{p}^{T}\left(\boldsymbol{z}^{-1}\right) \mathbf{G}_{p}(\boldsymbol{z})=\mathbf{I}$, which leads to a difficult factorization problem.

Vaidyanathan and Hoang provided a complete characterization of one-dimensional orthogonal filter banks via a lattice factorization [15](pp. 302-322). Kovačević and Vetterli extended this result to the 2-D case [12]. Using the lattice structure, the synthesis polyphase matrix can be represented as

$$
\mathbf{G}_{n}\left(z_{1}, z_{2}\right)=\mathbf{R}_{0} \prod_{j=1}^{n} \mathbf{D}_{j} \mathbf{R}_{j}
$$


where $\mathbf{R}_{j}$ is an arbitrary orthogonal matrix and $\mathbf{D}_{j}$ is a diagonal matrix. For convenience, we call $n$ the degree of the filter bank and let $G_{n}\left(z_{1}, z_{2}\right)$ denote the corresponding lowpass filter. For $\mathbf{D}_{j}$, its value depends on the parity of $j$ :

$$
\mathbf{D}_{j}=\left\{\begin{array}{ll}
\mathbf{D}\left(z_{1}\right) & \text { if } j \text { is odd } \\
\mathbf{D}\left(z_{2}\right) & \text { if } j \text { is even }
\end{array},\right.
$$

where

$$
\mathbf{D}(z)=\left(\begin{array}{cc}
1 & 0 \\
0 & z^{-1}
\end{array}\right) .
$$

The orthogonal matrix $\mathbf{R}_{j}$ can be represented by

$$
\mathbf{R}_{j}=\left(\begin{array}{cc}
\cos \theta_{j} & \sin \theta_{j} \\
-\sin \theta_{j} & \cos \theta_{j}
\end{array}\right) .
$$

For computation convenience, $\mathbf{R}_{j}$ can also be represented by

$$
\mathbf{R}_{j}=\frac{1}{\sqrt{1+a_{j}^{2}}}\left(\begin{array}{cc}
1 & a_{j} \\
-a_{j} & 1
\end{array}\right), \quad \text { where } a_{j} \in \mathbb{R} \bigcup\{ \pm \infty\},
$$

and $a_{j}=\tan \theta_{j}$. In what follows, we will use both representations in terms of convenience.

Orthogonal filter banks with lattice structures are easy to design and implement. Although this characterization of orthogonal filter banks is not complete, it provides enough freedom to design good filters.

\section{ORTHOGONAL FILTER BANKS WITH DIRECTIONAL VANISHING MOMENTS}

\subsection{Formulation}

Directional vanishing moments are vanishing moments along a specific direction. For example, if a 2-D filter $f\left(z_{1}, z_{2}\right)$ has $L$ th-order zero partial derivatives with respect to $z_{1}$ along the straight line $z_{1}=-1$, then it is said to have $L$ th-order horizontal vanishing moments. In this section, we focus on orthogonal filter banks with first-order directional vanishing moments (OFDVM).

Quincunx sampling has been extensively used in directional filter banks and contourlet transforms. For the 2-D quincunx sampling, its sampling matrix and the integer vectors in (1) can be written as

$$
\mathbf{D}=\left(\begin{array}{cc}
1 & 1 \\
1 & -1
\end{array}\right), \quad \text { and } \quad \boldsymbol{l}_{0}=\left(\begin{array}{l}
0 \\
0
\end{array}\right), \boldsymbol{l}_{1}=\left(\begin{array}{l}
1 \\
0
\end{array}\right)
$$

In terms of (1), the lowpass filter in the quincunx sampling can be computed as

$$
\begin{aligned}
G_{n}\left(z_{1}, z_{2}\right) & =\left(\begin{array}{ll}
1 & z_{1}
\end{array}\right) \mathbf{G}_{n}\left(z_{1} z_{2}, z_{1} z_{2}^{-1}\right)\left(\begin{array}{l}
1 \\
0
\end{array}\right) \\
& =\left(\begin{array}{ll}
1 & z_{1}
\end{array}\right) \mathbf{R}_{0} \prod_{j=1}^{n} \mathbf{D}_{j}^{\prime} \mathbf{R}_{j}\left(\begin{array}{l}
1 \\
0
\end{array}\right)
\end{aligned}
$$

where

$$
\mathbf{D}_{j}^{\prime}=\left\{\begin{array}{ll}
\mathbf{D}\left(z_{1}^{-1} z_{2}^{-1}\right) & \text { if } j \text { is odd } \\
\mathbf{D}\left(z_{1}^{-1} z_{2}\right) & \text { if } j \text { is even }
\end{array},\right.
$$

Imposing the first-order DVM condition, we have

$$
G_{n}\left(-1, z_{2}\right)=\left(\begin{array}{ll}
1 & -1
\end{array}\right) \mathbf{G}_{n}\left(-z_{2},-z_{2}^{-1}\right)\left(\begin{array}{l}
1 \\
0
\end{array}\right) \equiv 0, \quad \text { for } z_{2} \in \mathbb{R} .
$$


Table 1. Orthogonal filter banks with first-order directional vanishing moments of degree less than 3.

\begin{tabular}{l|l|l} 
Degree & Polyphase matrix & Parameters \\
\hline 0 & $\mathbf{R}_{0}$ & $\theta_{0}=m \pi-\pi / 4$ \\
\hline 1 & $\mathbf{R}_{0} \mathbf{D}\left(z_{1}\right) \mathbf{R}_{1}$ & $\theta_{0}=m \pi-\pi / 4, \theta_{1}=m \pi$ \\
& & $\theta_{0}=m \pi+\pi / 4, \theta_{1}=m \pi+\pi / 2$ \\
\hline 2 & $\mathbf{R}_{0} \mathbf{D}\left(z_{1}\right) \mathbf{R}_{1} \mathbf{D}\left(z_{2}\right) \mathbf{R}_{2}$ & $\theta_{1}=m \pi, \theta_{0}+\theta_{2}=m \pi-\pi / 4$ \\
& & $\theta_{2}=m \pi, \theta_{0}=m \pi+\pi / 4, \theta_{1}=m \pi+\pi / 2$
\end{tabular}

Therefore, the design problem of OFDVM is to find a set of rotation parameters $\left\{\theta_{0}, \theta_{1}, \cdots, \theta_{n}\right\}$ satisfying

$$
\left(\begin{array}{ll}
1 & -1
\end{array}\right) \mathbf{R}_{0} \prod_{j=1}^{n} \mathbf{D}\left(-z_{2}^{(-1)^{j+1}}\right) \mathbf{R}_{j}\left(\begin{array}{l}
1 \\
0
\end{array}\right) \equiv 0 .
$$

Condition (5) leads to nonlinear equations in terms of the rotation parameters, which are difficult to solve directly.

\subsection{Two Simple Cases}

In this subsection, we investigate how to design OFDVM with lowest degree, specifically, $n=0$ and $n=1$. The higher degree cases can be converted into $n=0$ and $n=1$ cases, as we will see in the next subsection.

1. 0th degree OFDVM.

In this case, $\mathbf{G}_{0}=\mathbf{R}_{0}$ and (5) becomes

$$
\begin{aligned}
0 & =\left(\begin{array}{ll}
1 & -1
\end{array}\right)\left(\begin{array}{cc}
\cos \theta_{0} & \sin \theta_{0} \\
-\sin \theta_{0} & \cos \theta_{0}
\end{array}\right)\left(\begin{array}{l}
1 \\
0
\end{array}\right) \\
& =\cos \theta_{0}+\sin \theta_{0} .
\end{aligned}
$$

Therefore,

$$
\theta_{0}=m \pi-\pi / 4, \quad \text { where } \quad m \in \mathcal{Z} .
$$

2. First degree OFDVM.

In this case,

$$
\mathbf{G}_{1}\left(-z_{2},-z_{2}^{-1}\right)=\mathbf{R}_{0} \mathbf{D}\left(-z_{2}\right) \mathbf{R}_{1} .
$$

Put (7) into (5), and then

$$
\left(\begin{array}{ll}
1 & -1
\end{array}\right) \mathbf{R}_{0} \mathbf{D}\left(-z_{2}\right) \mathbf{R}_{1}\left(\begin{array}{l}
1 \\
0
\end{array}\right) \equiv 0
$$

After simple manipulations,

$$
\cos \theta_{1}\left(\sin \theta_{0}+\cos \theta_{0}\right)+\sin \theta_{1}\left(\sin \theta_{0}-\cos \theta_{0}\right) z_{2}^{-1} \equiv 0,
$$

which leads to

$$
\theta_{0}=m \pi-\pi / 4, \theta_{1}=m \pi \quad \text { or } \quad \theta_{0}=m \pi+\pi / 4, \theta_{1}=m \pi+\pi / 2
$$

The design results for the two simple cases are given in Table 1. It can be seen that they share similar structure. 


\subsection{Reduction and Combination Characterization}

In this subsection, we show that it is possible to reduce the number of unknown parameters systematically for OFDVM of arbitrary degree. Moreover, the reduction continues until the design problem is reduced to that of 0th or first degree OFDVM.

THEOREM 1. Suppose $G_{n}\left(z_{1}, z_{2}\right)$ in (2) has first-order directional vanishing moments and $n>1$. Then at least one of $a_{1}, a_{2}, \cdots, a_{n}$ must be zero.

According to Theorem 1, there are two types of reduction. One is that one of the middle parameters is zero, and the second is that the last parameter is zero. For the first case, assume that $a_{l}=0,0<l<n$, and then $\mathbf{R}_{l}$ becomes an identity matrix, and

$$
\mathbf{D}_{l} \mathbf{R}_{l} \mathbf{D}_{l+1}=\mathbf{D}_{l} \mathbf{D}_{l+1}=\mathbf{D}\left(z_{1} z_{2}\right)
$$

Replacing $z_{1}$ and $z_{2}$ by $-z_{2}$ and $-z_{2}^{-1}$, respectively, we have

$$
\mathbf{D}_{l} \mathbf{R}_{l} \mathbf{D}_{l+1}=\mathbf{D}\left(\left(-z_{2}\right)\left(-z_{2}^{-1}\right)\right)=\mathbf{D}(1)=\mathbf{I} \text {. }
$$

Therefore, $\mathbf{D}_{l} \mathbf{R}_{l} \mathbf{D}_{l+1}$ is cancelled in (5), which generates a new lattice structure $\mathbf{R}_{l-1} \mathbf{R}_{l+1}$ in (5). Since

$$
\mathbf{R}_{l-1} \mathbf{R}_{l+1}=\left(\begin{array}{cc}
\cos \left(\theta_{l-1}+\theta_{l+1}\right) & \sin \left(\theta_{l-1}+\theta_{l+1}\right) \\
-\sin \left(\theta_{l-1}+\theta_{l+1}\right) & \cos \left(\theta_{l-1}+\theta_{l+1}\right)
\end{array}\right)
$$

they can be seen as one orthogonal matrix in (5) with the angle $\theta_{l-1}+\theta_{l+1}$. We call this process combination. Therefore, the rest of the design is just equivalent to that of $(n-2)$-th degree.

For the second case, $\mathbf{R}_{n}$ becomes an identity matrix, and $G_{n}\left(z_{1}, z_{2}\right)$ becomes $G_{n-1}\left(z_{1}, z_{2}\right)$. The design problem is the same as that of $n-1$ degree.

To sum up, the design problem of $n$th degree OFDVM is converted to that of either $(n-1)$-th degree or $(n-2)$-th degree OFDVM. In this process, there are two key components, reduction and combination. We can repeat this process until the final problem is either 0th degree or first degree. To illustrate this process, we give an example.

EXAMPLE 1. Find the solutions for the second degree OFDVM, which can be written as

$$
\mathbf{G}_{2}\left(z_{1}, z_{2}\right)=\mathbf{R}_{0} \mathbf{D}\left(z_{1}\right) \mathbf{R}_{1} \mathbf{D}\left(z_{2}\right) \mathbf{R}_{2}
$$

By Theorem 1, either $\theta_{1}$ or $\theta_{2}$ equals zero. The design problem is reduced to the following cases:

1. $\theta_{1}$ equals zero. The design problem is converted to that of the 0 th degree with the format

$$
\mathbf{G}_{0}\left(z_{1}, z_{2}\right)=\mathbf{R}_{02} \text {. }
$$

Therefore, the solution is

$$
\theta_{1}=m \pi, \theta_{0}+\theta_{2}=m \pi-\pi / 4
$$

2. $\theta_{2}$ equals zero. The design problem is converted to that of the first degree with the format

$$
\mathbf{G}_{1}\left(z_{1}, z_{2}\right)=\mathbf{R}_{0} \mathbf{D}\left(z_{1}\right) \mathbf{R}_{1}
$$

Therefore, the solution is

$$
\begin{aligned}
& \theta_{2}=m \pi, \theta_{0}=m \pi-\pi / 4, \theta_{1}=m \pi \quad \text { or } \\
& \theta_{2}=m \pi, \theta_{0}=m \pi+\pi / 4, \theta_{1}=m \pi+\pi / 2 .
\end{aligned}
$$

The solutions are summarized in Table 1. 


\subsection{Closed-Form Expression}

The closed-form solutions for arbitrary degree OFDVM can be generated iteratively by simple symbolic computation.

Denote that the solution of $n$th degree OFDVM is given by $s_{n}\left(\theta_{0}, \theta_{1}, \ldots, \theta_{n}\right)$. Two initial expressions are

$$
\begin{aligned}
& s_{0}\left(\theta_{0}\right)=\left\{\theta_{0}=m \pi-\pi / 4\right\}, \\
& s_{1}\left(\theta_{0}, \theta_{1}\right)=\left\{\theta_{0}=m \pi-\pi / 4, \theta_{1}=m \pi\right\} \bigcup\left\{\theta_{0}=m \pi+\pi / 4, \theta_{1}=m \pi+\pi / 2\right\} .
\end{aligned}
$$

For the first type of reduction, suppose $\theta_{l}, 0<l<n$ equals to zero. Then $\theta_{l-1}$ and $\theta_{l+1}$ are combined together, and the solution is determined by the solution of $(n-2)$-th degree OFDVM. In other words, the solution can be written as

$$
\left\{\theta_{l}=m \pi\right\} \bigcap s_{n-2}\left(\theta_{0}, \theta_{1}, \ldots, \theta_{l-2}, \theta_{l-1}+\theta_{l+1}, \theta_{l+2}, \ldots, \theta_{n}\right) .
$$

For the second type of reduction, $\theta_{n}$ equals to zero and the remaining $n-1$ parameters are determined by the solution of $(n-1)$-th degree OFDVM. In other words, the solution can be written as

$$
\left\{\theta_{n}=m \pi\right\} \bigcap s_{n-1}\left(\theta_{0}, \theta_{1}, \ldots, \theta_{n-1}\right) .
$$

To sum up, we have an iterative expression for the solution of $n$th degree OFDVM:

$$
\begin{aligned}
s_{n}\left(\theta_{0}, \theta_{1}, \ldots, \theta_{n}\right)= & \bigcup_{l=1}^{n-1}\left(\left\{\theta_{l}=m \pi\right\} \bigcap s_{n-2}\left(\theta_{0}, \theta_{1}, \ldots, \theta_{l-2}, \theta_{l-1}+\theta_{l+1}, \theta_{l+2}, \ldots, \theta_{n}\right)\right) \\
& \bigcup\left(\left\{\theta_{n}=m \pi\right\} \bigcap s_{n-1}\left(\theta_{0}, \theta_{1}, \ldots, \theta_{n-1}\right)\right),
\end{aligned}
$$

for $n>1$. The initial expressions are given in (10) and (11).

To illustrate this method, we give an example.

EXAMPLE 2. We consider the case when the degree is 3. By (14), we have

$$
\begin{aligned}
s_{3}\left(\theta_{0}, \theta_{1}, \theta_{2}, \theta_{3}\right)= & \left(\left\{\theta_{1}=m \pi\right\} \bigcap s_{1}\left(\theta_{0}+\theta_{2}, \theta_{3}\right)\right) \\
& \bigcup\left(\left\{\theta_{2}=m \pi\right\} \bigcap s_{1}\left(\theta_{0}, \theta_{1}+\theta_{3}\right)\right) \\
& \bigcup\left(\left\{\theta_{3}=m \pi\right\} \bigcap s_{2}\left(\theta_{0}, \theta_{1}, \theta_{2}\right)\right) .
\end{aligned}
$$

By (11),

$$
\begin{aligned}
& s_{1}\left(\theta_{0}+\theta_{2}, \theta_{3}\right)=\left\{\theta_{0}+\theta_{2}=m \pi-\pi / 4, \theta_{3}=m \pi\right\} \bigcup\left\{\theta_{0}+\theta_{2}=m \pi+\pi / 4, \theta_{3}=m \pi+\pi / 2\right\}, \\
& s_{1}\left(\theta_{0}, \theta_{1}+\theta_{3}\right)=\left\{\theta_{0}=m \pi-\pi / 4, \theta_{1}+\theta_{3}=m \pi\right\} \bigcup\left\{\theta_{0}=m \pi+\pi / 4, \theta_{1}+\theta_{3}=m \pi+\pi / 2\right\} .
\end{aligned}
$$

It is easy to verify that the last term in (15) is a subset of the union of first two terms. The solutions are summarized in Table 2.

In the above example, we find that the solutions due to the second type of reduction are a subset of the solutions due to the first type of reduction. In this case, we do not need to compute the solution due to the second type of reduction. The following theorem gives a simplified formula.

THEOREM 2. For $n>1$, the solution of nth degree orthogonal filter banks with directional vanishing moments can be simplified as

$$
s_{n}\left(\theta_{0}, \theta_{1}, \ldots, \theta_{n}\right)=\bigcup_{l=1}^{\lfloor n / 2\rfloor+1}\left(\left\{\theta_{l}=m \pi\right\} \bigcap s_{n-2}\left(\theta_{0}, \theta_{1}, \ldots, \theta_{l-2}, \theta_{l-1}+\theta_{l+1}, \theta_{l+2}, \ldots, \theta_{n}\right)\right),
$$

where $\lfloor n / 2\rfloor$ stands for the maximal integer that is not greater than $n / 2$. 
Table 2. Third degree orthogonal FIR filter banks with first-order directional vanishing moments. The polyphase matrix is $\mathbf{R}_{0} \mathbf{D}\left(z_{1}\right) \mathbf{R}_{1} \mathbf{D}\left(z_{2}\right) \mathbf{R}_{2} \mathbf{D}\left(z_{1}\right) \mathbf{R}_{3}$.

\begin{tabular}{l|l} 
Index & Parameters \\
\hline 1 & $\theta_{1}=m \pi, \theta_{0}+\theta_{2}=m \pi-\pi / 4, \theta_{3}=m \pi$ \\
2 & $\theta_{1}=m \pi, \theta_{0}+\theta_{2}=m \pi+\pi / 4, \theta_{3}=m \pi+\pi / 2$ \\
3 & $\theta_{2}=m \pi, \theta_{0}=m \pi-\pi / 4, \theta_{1}+\theta_{3}=m \pi$ \\
4 & $\theta_{2}=m \pi, \theta_{0}=m \pi+\pi / 4, \theta_{1}+\theta_{3}=m \pi+\pi / 2$
\end{tabular}

Table 3. Coefficients of the lowpass filter in Example $3 h\left[n_{1}, n_{2}\right]$.

\begin{tabular}{c|ccc}
$n_{1} \backslash n_{2}$ & 0 & 1 & 2 \\
\hline 0 & 0 & $-\frac{3}{32 \sqrt{2}}$ & $\frac{\sqrt{15}}{32 \sqrt{2}}$ \\
1 & $-\frac{\sqrt{15}}{32 \sqrt{2}}$ & $\frac{5}{32 \sqrt{2}}$ & 0 \\
2 & 0 & $\frac{\sqrt{15}}{16 \sqrt{2}}$ & $-\frac{\sqrt{15}}{16 \sqrt{2}}$ \\
3 & $\frac{\sqrt{15}}{16 \sqrt{2}}$ & $\frac{\sqrt{15}}{16 \sqrt{2}}$ & 0 \\
4 & 0 & $\frac{5}{32 \sqrt{2}}$ & $\frac{\sqrt{15}}{32 \sqrt{2}}$ \\
5 & $-\frac{\sqrt{15}}{32 \sqrt{2}}$ & $-\frac{3}{32 \sqrt{2}}$ & 0
\end{tabular}

\section{ORTHOGONAL FILTER BANKS WITH HIGHER-ORDER DVM}

We have proposed the complete characterization of orthogonal filter banks with first-order directional vanishing moments. Based on this characterization, we can further design orthogonal filter banks with higher-order DVM by imposing additional derivative constraints. We do not have closed-form expressions. However, the first-order DVM characterization has solved most unknowns and the number of additional equations is small and can be solved easily. In this section, we illustrate the design method by examples.

We find the minimal size orthogonal filter banks with second-order DVM. It is easy to verify that there is no solution for $n<6$. When $n=6$, the parameters are specified by

$$
\theta_{2}=m \pi, \theta_{4}=m \pi, \theta_{0}+\theta_{6}=m \pi-\pi / 4, \theta_{1}+\theta_{3}+\theta_{5}=m \pi
$$

It is difficult to solve the triangular equation directly. Therefore, we use the second expression for the orthogonal matrix. Accordingly, we convert (17) into a set of equations in $\left\{a_{j}\right\}$ :

$$
a_{2}=0, a_{4}=0, a_{0}=\frac{a_{6}+1}{a_{6}-1}, a_{1}=\frac{a_{5}+a_{3}}{a_{5} a_{3}-1} .
$$

The free variables are $a_{3}, a_{5}, a_{6}$. By solving the constraints imposed by the second-order DVM, we have

$$
\begin{aligned}
& a_{3}=\frac{2 a_{5}}{a_{5}^{2}-1}, \\
& a_{6}=\frac{6-2 a_{5}^{2}+\sqrt{35-26 a_{5}^{2}+3 a_{5}^{4}}}{1+a_{5}^{2}} .
\end{aligned}
$$

Therefore, we obtain a family of solutions with one degree of freedom, $a_{5}$.

EXAMPLE 3. When $a_{5}= \pm \sqrt{\frac{5}{3}}$, we have $35-26 a_{5}^{2}+3 a_{5}^{4}=0$ in (19). In this case, the resulting lowpass filter is given in Table 3, and its magnitude frequency response is shown in Fig. 2(a). Do also found this filter by brute force. Actually, Do also found that it is the minimal orthogonal filter with second-order directional vanishing moments. 
Table 4. Coefficients of the lowpass filter in Example $4 h\left[n_{1}, n_{2}\right]$.

\begin{tabular}{c|ccc}
$n_{1} \backslash n_{2}$ & 0 & 1 & 2 \\
\hline 0 & 0 & $\frac{3}{32 \sqrt{2}}$ & 0 \\
1 & $-\frac{3 \sqrt{7}}{32 \sqrt{2}}$ & 0 & 0 \\
2 & 0 & $-\frac{\sqrt{7}}{16 \sqrt{2}}$ & $\frac{3 \sqrt{7}}{32 \sqrt{2}}$ \\
3 & $\frac{3 \sqrt{7}}{16 \sqrt{2}}$ & $-\frac{21}{32 \sqrt{2}}$ & 0 \\
4 & 0 & $-\frac{21}{32 \sqrt{2}}$ & $-\frac{3 \sqrt{7}}{32 \sqrt{2}}$ \\
5 & $-\frac{3 \sqrt{7}}{32 \sqrt{2}}$ & $-\frac{\sqrt{7}}{16 \sqrt{2}}$ & 0 \\
6 & 0 & 0 & $\frac{3 \sqrt{7}}{32 \sqrt{2}}$ \\
7 & 0 & $\frac{3}{32 \sqrt{2}}$ & 0
\end{tabular}

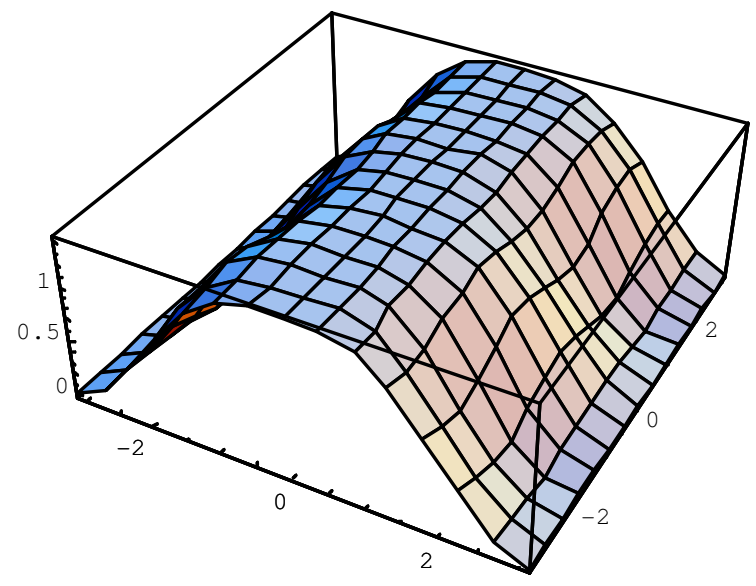

(a)

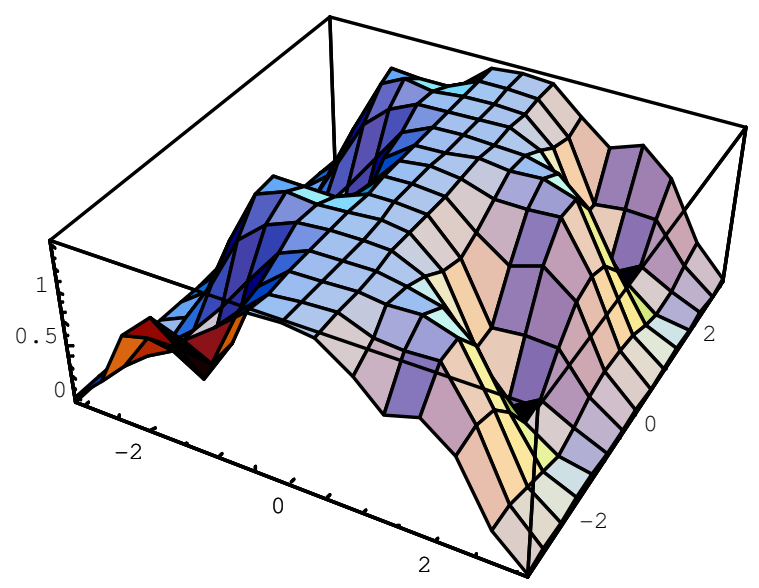

(b)

Figure 2. Magnitude frequency responses of two orthogonal lowpass filters with second-order directional vanishing moments: (a) Example 3. (a) Example 4.

EXAMPLE 4. When $a_{5}= \pm \sqrt{7}$, we also have $35-26 a_{5}^{2}+3 a_{5}^{4}=0$ in (19). In this case, the resulting lowpass filter is given in Table 4, and its magnitude frequency response is shown in Fig. 2(b). To the best of our knowledge, it is a new filter.

EXAMPLE 5. We can also impose some additional point vanishing moments by

$$
\left.\frac{\partial^{2} H\left(z_{1}, z_{2}\right)}{\partial z_{2}^{2}}\right|_{(-1,-1)}=0 .
$$

There are two solutions:

$$
\begin{aligned}
& a_{5}=1.144928297722449, \\
& a_{5}=9.495665386679022 .
\end{aligned}
$$

The magnitude frequency responses of the resulting lowpass filters are given in in Fig. 3. They have good diamond shape.

\section{CONCLUSION}

We have proposed a method based on lattice structures to design orthogonal FIR filter banks with directional vanishing moments. We consider orthogonal filter banks generated by lattice structures. For this class of filter 

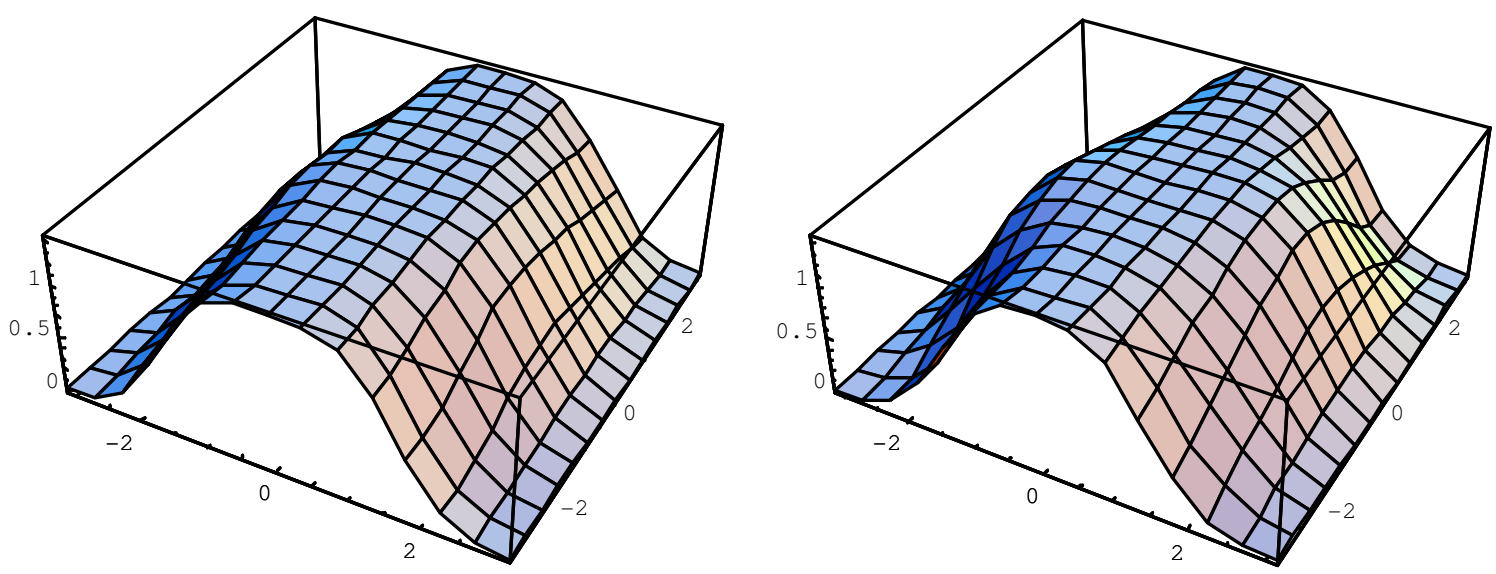

Figure 3. Magnitude frequency responses of two orthogonal lowpass filters in Example 5. They have second-order directional vanishing moments and one additional point vanishing moment.

banks, we find a complete closed-form solution for the directional-vanishing-moment condition. It also simplifies the design problem for orthogonal filter banks with higher-order directional vanishing moments. We illustrate our design method with examples.

\section{REFERENCES}

1. R. A. DeVore, B. Jawerth, and B. J. Lucier, "Image compression through wavelet transform coding," IEEE Transactions on Information Theory, Special Issue on Wavelet Transforms and Multiresolution Signal Analysis 38, pp. 719746, March 1992.

2. M. Vetterli, "Wavelets, approximation and compression," IEEE SP Mag., pp. 59-73, Sep. 2001.

3. I. Daubechies, "Orthonormal bases of compactly supported wavelets," Commun. on Pure and Appl. Math. 41, pp. 909-996, November 1988.

4. R. H. Bamberger, The Directional Filter Bank: A Multirate Filter Bank for the Directional Decomposition of Images. $\mathrm{PhD}$ thesis, Georgia Institute of Technology, 1990.

5. E. P. Simoncelli, W. T. Freeman, E. H. Adelson, and D. J. Heeger, "Shiftable multiscale transforms," IEEE Trans. Inform. Th. 38, pp. 587-607, March 1992.

6. F. G. Meyer and R. R. Coifman, "Brushlets: a tool for directional image analysis and image compression," Journal of Appl. and Comput. Harmonic Analysis 5, pp. 147-187, 1997.

7. D. L. Donoho, "Wedgelets: nearly-minimax estimation of edges," Ann. Statist. 27, pp. 859-897, 1999.

8. E. J. Candès and D. L. Donoho, "Curvelets - a suprisingly effective nonadaptive representation for objects with edges," in Curve and Surface Fitting, A. Cohen, C. Rabut, and L. L. Schumaker, eds., Vanderbilt University Press, (Saint-Malo), 1999.

9. M. N. Do and M. Vetterli, "The contourlet transform: An efficient directional multiresolution image representation," IEEE Trans. Image Proc., 2005. to appear, http://www.ifp.uiuc.edu/〜minhdo/publications.

10. B. A. Olshausen and D. J. Field, "Emergence of simple-cell receptive field properties by learning a sparse code for natural images," Nature (381), pp. 607-609, 1996.

11. A. L. Cunha and M. N. Do, "Biorthogonal two-channel filter banks with directional vanishing moments: Characterization, design and applications," IEEE Trans. Image Processing , submitted. http://www.ifp.uiuc.edu/ ${ }^{\sim}$ minhdo/publications.

12. J. Kovačević and M. Vetterli, "Nonseparable multidimensional perfect reconstruction filter banks and wavelet bases for $\mathcal{R}^{n}$," IEEE Transactions on Information Theory, Special Issue on Wavelet Transforms and Multiresolution Signal Analysis 38, pp. 533-555, March 1992.

13. D. Stanhill and Y. Y. Zeevi, "Two-dimensional orthogonal wavelets with vanishing moments," IEEE Trans. Signal Proc. 44, pp. 2579-2590, October 1996.

14. J. Zhou and M. N. Do, "Two-dimensional orthogonal filter banks with directional vanishing moments," IEEE Trans. Signal Proc. . to be submitted.

15. P. P. Vaidyanathan, Multirate Systems and Filter Banks, Prentice-Hall, 1993. 